\title{
A peregrinação das gestantes no Município do Rio de Janeiro: perfil de óbitos e nascimentos
}

\author{
THE PEREGRINATION OF THE PREGNANTS IN RIO DE JANEIRO CITY: \\ DEATHS AND BIRTHS PROFILE
}

\section{LA PEREGRINACIÓN DE LAS GESTANTES EN LO MUNICIPIO DEL RIO DE JANEIRO: PERFIL DE OBITOS Y NACIMIENTO}

\section{Enirtes Caetano Prates Melo', Virginia Maria de Azevedo Oliveira Knupp², Rejane Burlandi de Oliveira ${ }^{3}$, Teresa Tonini ${ }^{4}$}

\begin{abstract}
1 Enfermeira. Doutora em Ciências pela Escola Nacional de Saúde Pública, FIOCRUZ. Professora Adjunto do Departamento de Enfermagem em Saúde

Pública da Escola de Enfermagem Alfredo Pinto, Universidade Federal do Estado do Rio de Janeiro (UFRJ). Rio de Janeiro, RJ, Brasil.

2 Graduanda de Enfermagem da Escola de Enfermagem Alfredo Pinto, Universidade Federal do Estado do Rio de Janeiro (UFRJ). Bolsista PIBIC/CNPq. Rio de Janeiro, RJ, Brasil.

virgula_knupp@ yahoo.com.br

3 Enfermeira. Doutora em Saúde Coletiva pelo Instituto de Medicina Social, Univer sidade Estadual do

Rio de Janeiro (UERJ).

Professora Adjunto

do Departamento de Enfermagem Fundamental da Escola de Enfermagem Alfredo

Trata-se de um estudo ecológico. Objetivou-se analisar as trajetórias dos nascimentos no município do Rio de Janeiro e identificar a relação entre oferta de serviços de saúde e fluxo de gestantes entre local de residência e a maternidade. Foram utilizados dados dos Sistemas de Informação sobre Mortalidade (SIM) e Nascidos Vivos (SINASC) de 2004. O processamento e mapeamento dos dados foram feitos através do programa TabWin. O SINASC processou 99.042 declarações de nascidos vivos e o SIM processou 1.318 declarações de óbitos em menores de um ano no Município do Rio de Janeiro. Concluiuse que a possibilidade de intervenção no perfil da mortalidade infantil e materna desloca-se cada vez mais para a esfera dos serviços de saúde, e o acesso à assistência de qualidade tem papel fundamental na determinação da mortalidade. Deve-se investigar a existência de desigualdades no acesso a tais serviços.
\end{abstract} Pinto, Universidade Federal do Estado do Rio de Janeiro (UFRJ). Rio de Janeiro, RJ,

Brasil.

4 Enfermeira. Doutora em Saúde Coletiva pela Universidade Federal do Estado do Rio de Janeiro

(UFRJ). Professor Adjunto da UFRJ. ttonini@terra.com.br

\section{RESUMO}

\section{DESCRITORES}

Enfermagem em saúde pública. Mortalidade infantil.

Saúde materno-infantil.

Sistemas de Informação.

Distribuição espacial da

população.

\section{ABSTRACT}

This ecological study presented analyze of the birth pathways in the Rio de Janeiro City and identification of the relationship between the health services offer and pregnant flow between their households and the maternity. It was used the data available in the Mortality Information System (MIS) and Live Birth Information System (LBIS) in 2004. The TabWin program processed the data and drew the maps. The LBIS processed 99,042 declarations of live birth and MIS processed 1,318 declarations of deaths in less than one year old in the Rio Janeiro City. The conclusion was that the possibility of intervention in the infantile and maternal mortality profile has happened at the health services, and the access to the quality assistance has fundamental hole in the mortality determination. The existence of access inequalities to the services must be investigated.

\section{KEY WORDS}

Public health nursing.

Intant mortality.

Maternal and child health.

Information Systemes.

Residence characteristics.

\section{RESUMEN}

Tratase de un estudio ecológico que tiene como objetivo analizar las trayectorias de los nacimientos en lo Municipio del Rio de Janeiro y identificar la relación entre oferta de servicios de la salud y del flujo de gestantes entre el lugar de la residencia y la maternidad. Fueron utilizados los datos de los Sistemas de la Información sobre Mortalidad (SIM) y Nacidos Vivos (SINASC) de 2004. El proceso y el mapeamento de los datos fueron hechos a través del programa TabWin. El SINASC procesó 99.042 declaraciones de nacidos vivos y el SIM procesó 1.318 declaraciones de óbitos en menores de un año en lo Municipio del Rio de Janeiro. Concluyese que la posibilidad de intervención en el perfil de la mortalidad infantil y materna dislocase cada vez más para la esfera de los servicios de la salud, y el acceso a la asistencia de calidad tiene papel fundamental en la determinación de la mortalidad, débese, investigar la existencia de desigualdades en el acceso a tales servicios.

\section{DESCRIPTORES}

Enfermería en salud pública. Mortalidad infantil.

Salud materno-infantil.

Sistemas de Información.

Distribución espacial de la población. 


\section{INTRODUÇÃO}

Uma questão fundamental para o planejamento do setor saúde é a distribuição espacial dos serviços e de sua clientela. Os serviços de saúde estão inevitavelmente concentrados em determinados lugares e, conseqüentemente, são mais acessíveis às pessoas que deles estão mais próximas do que àquelas que estão mais distantes deles ${ }^{(1)}$. A identificação das redes alerta para problemas de acesso, identificando áreas com poucas opções, configurando pontos de estrangulamento, ou oportunidades de desconcentração e regionalizações alternativas, e a classificação dos fluxos segundo a estrutura da rede fornece rico material de investigação.

Esta pesquisa, financiada através do Edital MCT/CNPq/ MS - $\mathrm{n}^{\circ} 036 / 2004$, esteve voltada para dois problemas específicos: a morbi-mortalidade infantil e a mortalidade materna. Os coeficientes de mortalidade infantil (CMI) e de mortalidade materna são reconhecidos não só como indicadores das condições de saúde da população, mas também como variáveis que refletem as condições gerais de vida, sendo consideradas sínteses da qualidade de vida e do nível de desenvolvimento de uma população.

Observou-se uma redução expressiva da mortalidade infantil na última década: em torno de 38\%. No Município do Rio de Janeiro o CMI situa-se em torno de 15 óbitos por mil nascidos vivos, com quase $70 \%$ das mortes ocorridas nos primeiros 28 dias de vida, mas ainda, com expressiva representação das causas evitáveis $^{(2)}$. Estudo realizado ${ }^{(3)}$ mostrou que os componentes da mortalidade infantil apresentaram distribuição espacial dispersa, sem uma rela-

A mudança no perfil da mortalidade infantil e a possibilidade de intervenção parecem deslocar-se cada vez mais para a esfera dos serviços de saúde, especialmente os médicos assistenciais. ção direta com o perfil socioeconômico. A mudança no perfil da mortalidade infantil e a possibilidade de intervenção parecem deslocar-se cada vez mais para a esfera dos serviços de saúde, especialmente os médico-assistenciais.

O componente pós-neonatal da mortalidade infantil apresentou uma queda expressiva por ser mais vulnerável às melhorias das condições de vida e intervenções no setor saúde, tais como: imunizações, tratamento adequado de diarréias e infecções respiratórias; incentivo ao aleitamento materno e melhoria nas condições de saneamento básico. Já o componente neonatal expressa diretamente a assistência durante o pré-natal, ao parto e a período neonatal, refletindo a cobertura e a qualidade dos serviços de saúde de uma região(4).

Fatores sócio-demográficos culturais maternos (idade, escolaridade, renda e estado civil) têm marcada influência no acesso aos serviços de atendimento a gravidez e ao nascimento, mesmo nos países desenvolvidos que apresentam melhores indicadores globais de saúde materno-infantil, mostrando que as possibilidades de utilização dos recursos
A área de estudo é o Município do Rio de Janeiro no ano de 2004, administrativamente dividido em 153 bairros, agregados em 30 Regiões Administrativas (RA). As RAs compõem cinco Áreas de Planejamento (AP). Estas áreas mantêm em comum, além da proximidade geográfica, o perfil socioeconômico e de acesso aos serviços de saúde. A AP 1 configura a zona mais antiga e central da cidade. As APs 2.1 e 2.2 correspondem respectivamente à Zona Sul e Zona Norte. As APs 3.1, 3.2 e 3.3, correspondem ao subúrbio da Central e Leopoldina, além da Ilha do Governador. As APs 4, 5.1, 5.2 e 5.3 correspondem à Zona Oeste.

A taxa de mortalidade infantil foi calculada para $\mathrm{o}$ ano de 2004 e representada no mapa de fluxo. No caso especificamente da dinâmica espacial dos nascimentos optou-se por concentrar a análise do mapa de fluxo em 2004. A análise da distribuição percentual dos óbitos no período de 1979 a 2004 foi utilizada para visualizar a evolução da mortalidade infantil e seus componentes ao longo do tempo.

Os mapas de fluxos com flechas permitem identificar o volume de tráfego entre local de residência (AP de residên- 
cia) e de assistência (AP de ocorrência), desde que identificados nos Sistemas de Informação em Saúde. A largura de cada flecha é proporcional ao fluxo. O padrão das setas permite verificar as dinâmicas percorridas em linha reta pela população na busca pela assistência, bem como identificar anomalias e particularidades neste fluxo, áreas que concentram os serviços de saúde e áreas com vazios sanitários. Neste estudo trabalhou-se somente com a origem e os destinos finais, uma vez que o percurso não está disponível nos SIS.

O georeferenciamento dos nascimentos e dos óbitos foi feito com base nas variáveis bairro de residência e endereço do estabelecimento de saúde onde ocorreu o nascimento e o óbito. O processamento e mapeamento dos dados foram feitos através do programa de código aberto TAB para Windows - TabWin, desenvolvido pelo DATASUS.

\section{RESULTADOS}

No município praticamente $100 \%$ dos partos são hospitalares e se concentram basicamente na rede própria do Sistema Único de Saúde (SUS), com mais de 70\% dos nascimentos. Em 2004 O SINASC processou 99.042 declarações de nascidos vivos no Município do Rio de Janeiro; dessas crianças, $82 \%$ (81.326) residiam no município. Os nascimentos de 2004 ocorreram em 98 estabelecimentos hospitalares, 30 dos quais atendiam pelo Sistema Único de Saúde.

A zona oeste (Jacarepaguá, Bangu, Campo Grande e Santa Cruz) apresenta a maior proporção de nascimentos. A localização geográfica dos estabelecimentos de saúde que atendem ao parto define uma distribuição espacial dos nascimentos extremamente desigual, alternado padrões de escassez em algumas áreas (Penha, Vigário Geral, Realengo, Tijuca) e excesso em outras (Madureira e Centro) (Figura 1). Nos nascimentos vinculados ao SUS observa-se, de um modo geral, que a utilização dos serviços se reduz à medida que aumentam as distâncias a serem percorridas pelos que demandam atendimento.

Estratificando-se os fluxos de nascimentos segundo o tipo de parto observa-se entre os partos cesáreos, de um modo geral, o mesmo padrão de deslocamento identificado na rede privada (Figura 2). Nos nascimentos vinculados ao SUS observa-se, de um modo geral, que a utilização dos serviços se reduz à medida que aumentam as distâncias a serem percorridas pelos que demandam atendimento. Esse padrão não se mantém fora do SUS, onde a distância percorrida pela gestante não parece representar um obstáculo capaz de se interpor entre a busca e a obtenção de serviços.

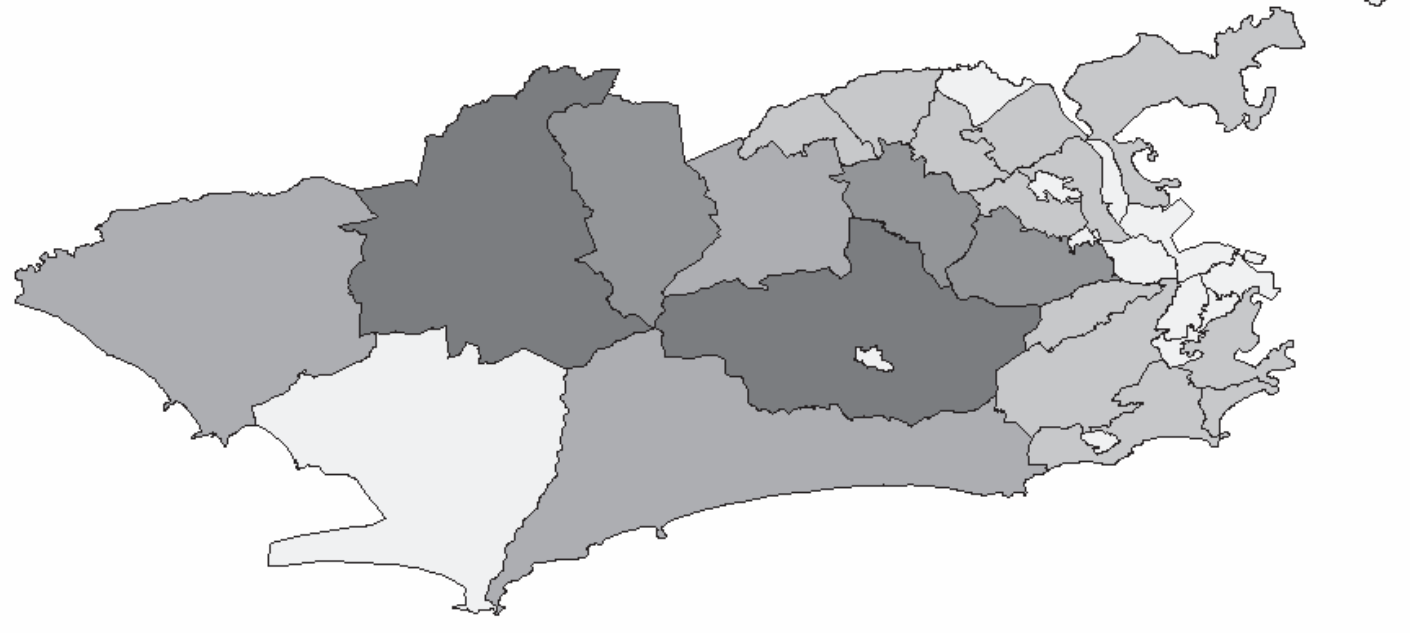

Figura 1 - Distribuição espacial dos nascimentos no Município do Rio de Janeiro, 2004 


\section{Parto Vaginal}
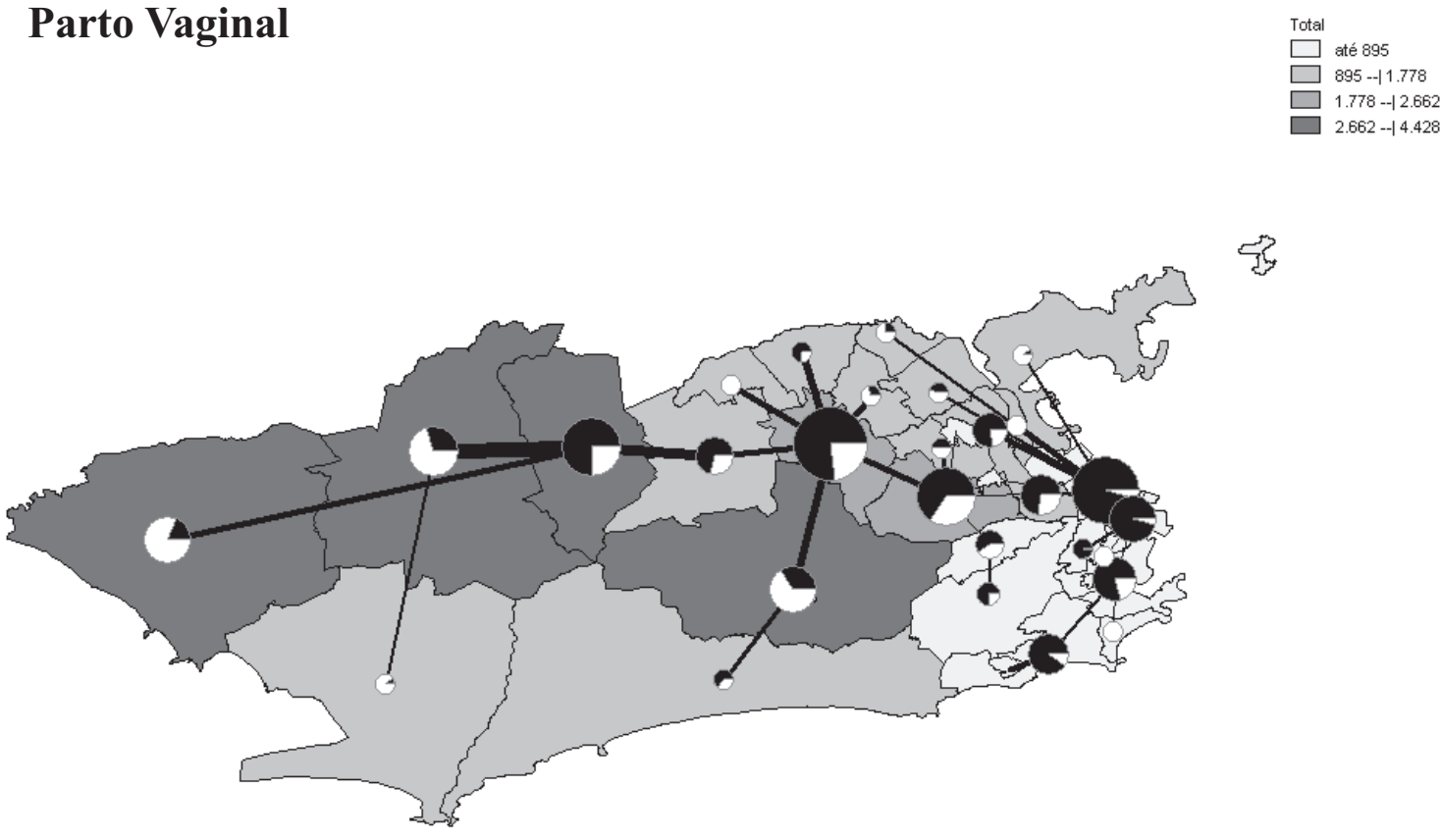

Fluxo Dominante (maior Fluxo na origem) + 1 pixel de largura a cada 135 casos

\section{Parto Cesáreo}
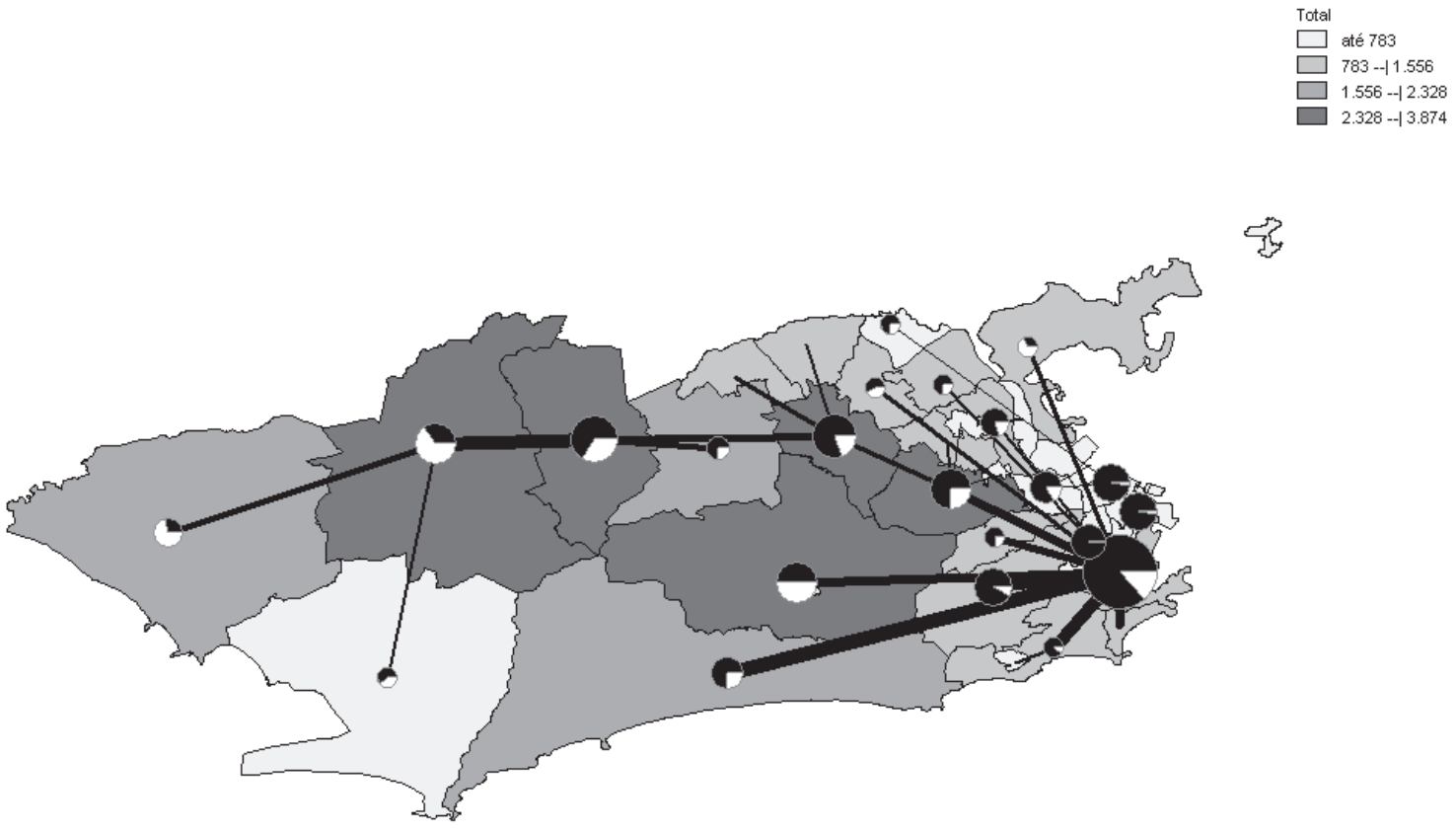

तु?

$\%$ de nascimentos na própria RA

Fluxo Dominante (maior Fluxo na origem)

$\%$ de nascimentos fora da RA

+1 pixel de largura a cada 95 casos

Figura 2 - Fluxo dos nascimentos segundo tipo de parto, Município do Rio de Janeiro, 2004 
O Sistema de Informações sobre Mortalidade (SIM) processou 1.318 declarações de óbitos em menores de um ano residentes no Município do Rio de Janeiro em 2004. No período de 1979 a 2004, verificou-se uma redução significante da mortalidade infantil, principalmente, o componente pósneonatal. Três grandes aglomerados com altas taxas de óbito infantil foram identificados (acima de 20/1000 NV), dos quais dois apresentam maior destaque (Figura 3). O primeiro, localizado na Zona Oeste, é composto pelas Regiões Administrativas (RAs) de Bangu, Santa Cruz, Campo Grande e Barra da Tijuca. O segundo aglomerado localizado na região central da cidade e composto pelas RAs do Centro, Portuária e São Cristóvão. O terceiro aglomerado, localizado no subúrbio da Leopoldina, se apresentou mais disperso.
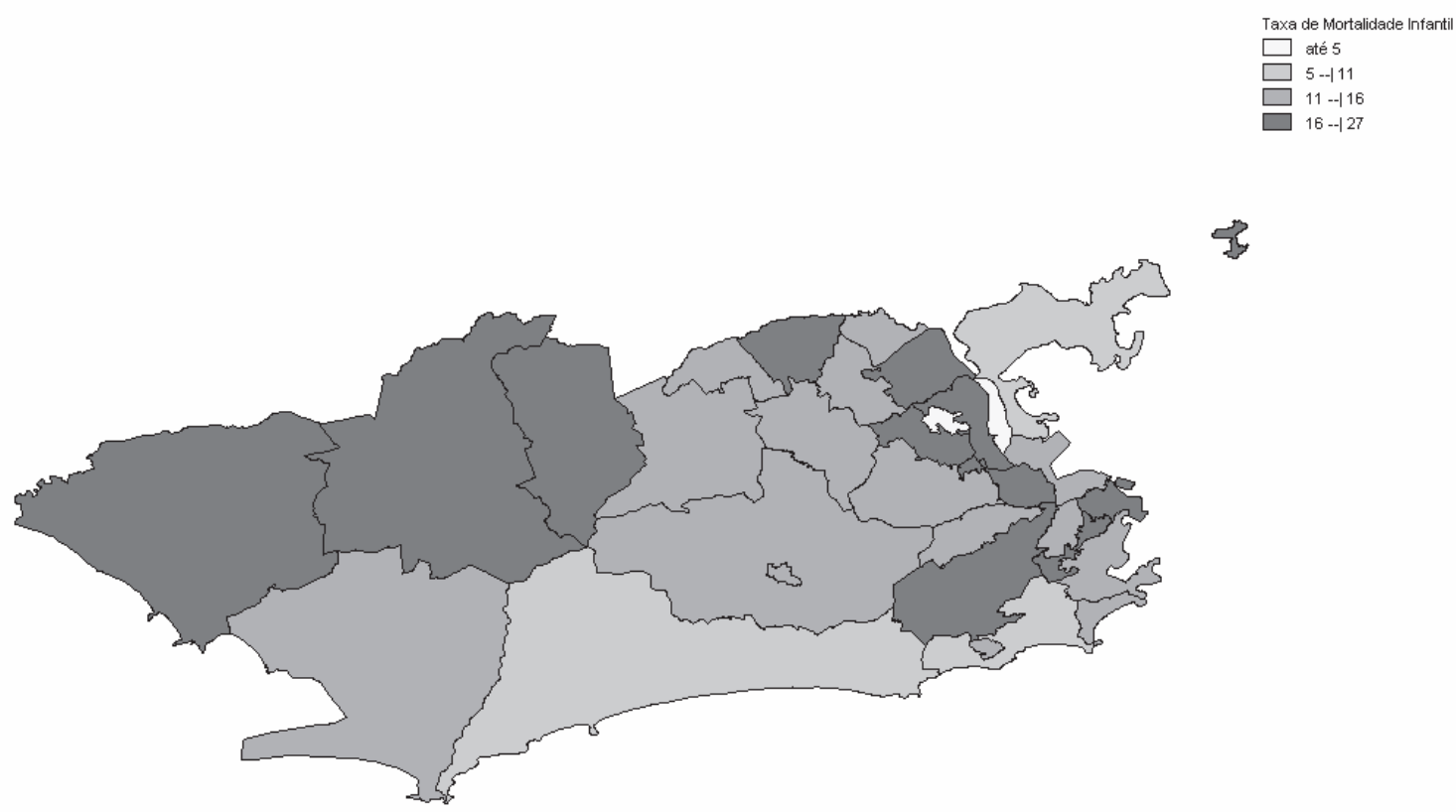

Figura 3 - Distribuição espacial da Taxa de Mortalidade Infantil no Município do Rio de Janeiro, 2004

No estudo dos fluxos de óbitos em menores de 1 ano entre a região administrativa de residência da mãe e de ocorrência do óbito em 2004 destacou-se um deslocamento no sentido oeste-leste, ou seja, da Zona Oeste em direção a região mais central da cidade, onde se concentram os serviços de saúde. Observa-se ainda que, na maioria das áreas, os fluxos dentro da própria região administrativa são muito menores se comparados aos inter-região administrativa.

\section{DISCUSSÃO}

O Rio de Janeiro agrega um padrão extremamente heterogêneo no que se refere ao processo de ocupação e à distribuição geográfica da população. Áreas remanescentes de floresta se alternam com áreas densamente povoadas; áreas consideradas nobres coexistem, lado a lado, com os estratos mais pobres da população. Essa composição traz para a cidade uma organização sócio-econômica espacial marcada por contrastes.

A localização geográfica dos estabelecimentos de saúde define uma distribuição desigual dos leitos obstétricos, al- ternando padrões de escassez em algumas áreas (periferia da cidade) e excesso em outras (Centro). Este padrão gera um grande fluxo de gestantes em busca de atendimento. Ao percorrer grandes distâncias torna-se mais difícil o acesso aos serviços e, principalmente, a adequação de suas necessidades aos serviços oferecidos ${ }^{(5)}$.

Os padrões espaciais observados no deslocamento das gestantes na estratificação segundo tipo de parto, estado civil, escolaridade e raça foram compatíveis aos padrões verificados nos indicadores de saúde relacionados à assistência materno-infantil, onde a precariedade das maternidades, assim como os fatores políticos, biológicas, sociais e culturais, contribui para a peregrinação das gestantes. A incorporação do elemento geográfico pode apoiar, neste caso, o redirecionamento de ações de saúde, principalmente em áreas onde se verifica maior exclusão social.

As dificuldades encontradas pelos clientes no uso dos serviços de saúde, isto é, na atitude de procurá-los, obter acesso e se beneficiar com o atendimento recebido, refletem as características da oferta dos serviços obstétricos que cada sociedade disponibiliza para seus membros. O uso dos 
serviços de saúde compreende todo contato direto (consultas médicas, consultas de enfermagem, consultas e hospitalizações) ou indireto (realização de exames preventivos e diagnósticos) com os serviços de saúde. Enquanto que, o processo de utilização dos serviços de saúde é resultante da interação do comportamento do cliente que procura atendimento e do profissional que o direciona dentro do sistema de saúde ${ }^{(6)}$.

O padrão da mortalidade infantil no Município do Rio de Janeiro é majoritariamente neonatal, portanto não é possível discutir estratégias para sua redução sem destacar o papel fundamental dos serviços de saúde através de uma adequada assistência pré-natal, ao parto e um adequado cuidado imediato com o recém-nascido. O mapeamento do fluxo dos óbitos infantis só permite identificar o destino final da clientela, não demonstrando o percurso/peregrinação das mães que buscam assistência para seus filhos. Observa-se que os deslocamentos identificados nos mapas de fluxo para a mortalidade são maiores se comparados aos identificados no mapa de fluxo de nascimentos.

\section{REFERENCIAS}

1. Lovett A, Haynes R, Sünnenberg G, Gale S. Car travel time and accessibility by bus to general practitioner services: a study using patient registers and GIS. Soc Sci Med. 2002;55(1): $97-111$

2. Leal MC, Gama SGN. Perinatalidade no Rio de Janeiro. Cad Saúde Pública. 2004;20 Supl 1:S4-5.

3. Campos TP, Carvalho MS. Assistência ao parto no Município do Rio de Janeiro: perfil das maternidades e o acesso da clientela. Cad Saúde Pública. 2000;16(2):411-20.
Os mapas de fluxos permitiram identificar o volume de tráfico entre local de residência e de assistência. O padrão das setas permitiu verificar as dinâmicas percorridas em linha reta pela população na busca pela assistência, bem como identificar anomalias e particularidades neste fluxo.

\section{CONCLUSÃO}

A identificação das redes que conectam os serviços e a população por eles atendida sinaliza áreas com poucas opções, configurando pontos de estrangulamento, ou oportunidades de desconcentração e regionalizações alternativas. A visualização dessas relações no território vem contribuir para a formulação das ações, relacionadas em particular às políticas públicas, mais integradas e efetivas, principalmente em áreas onde se verifica maior exclusão social.

A enfermagem exerce papel fundamental na visualização dessas relações no território e vem contribuir para a formulação das ações, relacionadas em particular às políticas públicas, mais integradas e efetivas, principalmente em áreas onde se verifica maior exclusão social.

4. Lansky S, França E, Leal MC. Mortalidade perinatal e evitabilidade: revisão de da literatura. Rev Saúde Pública. 2002;36 (6):759-72.

5. Menezes DSM, Leite IC, Schramm JMA, Leal MC. Avaliação da peregrinação anteparto numa amostra de puérpera no Município do Rio de Janeiro, Brasil, 1999/2001. Cad Saúde Pública. 2006;22(3):553-9.

6. Travassos C, Martins M. Uma revisão sobre os conceitos de acesso e utilização de serviços de saúde. Cad Saúde Pública. 2004;20 Supl 2:S190-8. 